\title{
Apelin protects against sepsis-induced cardiomyopathy by inhibiting the TLR4 and NLRP3 signaling pathways
}

\author{
QIANCHENG LUO ${ }^{1 *}$, GUORONG LIU $^{1 *}$, GUO CHEN $^{1}$, DONGFENG GUO ${ }^{1}$, \\ LEI XU ${ }^{1}$, MIN HANG ${ }^{1}$ and MINGMING JIN ${ }^{2}$ \\ ${ }^{1}$ Department of Emergency Medicine, Shanghai Pudong New Area Gongli Hospital; ${ }^{2}$ Department of Clinical Laboratory, \\ Shanghai Gongli Hospital, The Second Military Medical University, Shanghai 200135, P.R. China
}

Received December 7, 2017; Accepted May 2, 2018

DOI: $10.3892 /$ ijmm.2018.3665

\begin{abstract}
The mechanism underlying sepsis-induced cardiomyopathy (SICM) remains unclear. The aim of the present study was therefore to illuminate the mechanisms and effects of apelin on SICM, using both patient clinical features and a sepsis rat model. A total of 73 adult patients with or without sepsis were analyzed. Male rats were used to generate the sepsis model through cecal ligation and puncture (CLP). The clinical analysis results demonstrated that sepsis induced cardiac dysfunction, including a decrease of left ventricular end-diastolic dimension, fractional shortening, ejection fraction, left ventricular end-systolic dimension, and stroke volume, compared with healthy controls. In addition, the results demonstrated that white blood cell count and inflammatory cytokine expression increased in sepsis patients compared with healthy controls. ELISA analyses revealed that apelin was upregulated following sepsis. The animal model study demonstrated that rats treated with apelin had significantly reduced mortality and suppressed sepsis-induced myocardial damage and inflammatory responses, through suppression of activation of the Toll-like receptor 4 (TLR4) and NLR family pyrin domain containing 3 (NLRP3) signaling pathways. Taken together, the present results suggested that apelin had a protective effect against sepsis-induced cardiac impairment by attenuating TLR4 and NLRP3 signaling-mediated inflammatory responses.
\end{abstract}

Correspondence to: Dr Mingming Jin, Department of Clinical Laboratory, Shanghai Gongli Hospital, The Second Military Medical University, 219 Miao-Pu Road, Shanghai 200135, P.R. China E-mail: asdjinmingming@126.com

Dr Min Hang, Department of Emergency Medicine, Shanghai Pudong New Area Gongli Hospital, The Second Military Medical University, 219 Miao-Pu Road, Shanghai 200135, P.R. China

E-mail: 13817577126@139.com

*Contributed equally

Key words: apelin, sepsis, cardiomyopathy, Toll-like receptor 4, NLR family pyrin domain containing 3

\section{Introduction}

Sepsis is a severe clinical syndrome resulting from a systemic host response to infection. Septic-induced multiple organ dysfunctions or shock are usually the main causes of death in intensive care unit (ICU) patients $(1,2)$. Sepsis-induced cardiomyopathy (SICM) is attributed to various mechanisms, including mitochondrial dysfunction (3), alteration of expression of nitric oxide synthase (4), and inflammatory cytokine overexpression-induced cardiomyocyte (CM) apoptosis (5-7). Increasing evidence has demonstrated that there are close links between inflammation and innate immune response, which promote the progression of SICM (8-10). Inflammation involving inflammasome activation, mediated by NLR family pyrin domain containing 3 (NLRP3) and Toll-like receptor 4 (TLR4), has recently been established as an important mechanism regulating cardiac dysfunction (11). TLR4 activation induces nuclear factor (NF)- $\kappa \mathrm{B}$ mediated proinflammatory cytokine expression (12). Additionally, inflammatory responses are amplified with the induction of NLPR3 in the heart during SICM (13-15). The NLRP3 inflammasome, as a member of the NOD-like receptor (NLR) family, is comprised of apoptosis-associated speck-like protein (ASC), caspase-1 and NLRP3. Previous studies have reported that the NLRP3 region, not only controls pro-inflammatory cytokine production, but is also associated with the pathogenesis of inflammatory diseases (16-18).

Apelin is a peptide hormone, which is widely expressed in various tissues, and was recently identified as an endogenous ligand for a G-protein-coupled receptor $(19,20)$. Apelin has an important role in regulating phosphoinositide 3-kinase/Akt signaling-mediated angiectasis, inflammatory responses and cell proliferation (21). A recent study has also reported that anti-inflammatory effects were regulated by apelin/NF- $\kappa \mathrm{B}$ signaling (22).

In the present clinical study, apelin was demonstrated to be upregulated in SICM and the myocardial function was impaired with an enhanced inflammatory response following sepsis induction. To elucidate the protective mechanism of apelin, a cecal ligation and puncture (CLP)-induced sepsis-induced myocardial injury model was established in rats. The TLR4 and NLRP3 signaling pathway-related protein levels were then measured by western blot analysis, and the 
inflammatory factor levels were analyzed by ELISA. The present study identified potential new targets for the clinical treatment of sepsis.

\section{Materials and methods}

Subjects. A total of 73 subjects were recruited from Pudong New Area Gongli Hospital (Shanghai, China) from April 2016 to June 2017. The normal control subjects $(n=34)$ that were included in this study, had no family history of sepsis or other chronic diseases, were free of any major organ disease, and had a stable body weight for at least 1 year. In the ICU, sepsis was defined as an acute change in the total Sequential Organ Failure Assessment score $\geq 2$ points as a result of the infection $(n=39)$. The definition of sepsis-induced cardiomyopathy (SICM) was based on an ejection fraction (EF) $<50 \%$ and a $\geq 10 \%$ decrease compared with the baseline EF that recovered within 2 weeks (7). If the baseline was unknown, the baseline was defined as an increase of $>10 \%$ compared with the initial EF assessed on admission. The EF improving to baseline within 2 weeks was the definition of recovery. Inotropic agents, such as dobutamine and epinephrine, were not used prior to transthoracic echocardiography assessment. All clinical data were gathered from the electronic medical records written by residents or attending physicians of the emergency and critical care departments. The clinical characteristics of the patients are listed in Table I. To identify the relative expression of apelin in sepsis, $2 \mathrm{ml}$ plasma samples from each sepsis patient or healthy volunteer were collected. An informed consent was signed by each participant.

Sepsis model and treatment. Male Sprague Dawley rats (age, 7-8 weeks old; weight, 200-250 g) were provided by Shanghai SLAC Laboratory Animal Co. (Shanghai, China). Rats were provided with irradiated food, free access to sterile acidified water, and were housed in individual micro-isolators. For sepsis induction, animals were anesthetized by intraperitoneal injection of $2 \%$ sodium pentobarbital in saline $(40 \mathrm{mg} / \mathrm{kg}$; Sigma-Aldrich; Merck KGaA, Darmstadt, Germany) and placed on a warming pad $\left(37^{\circ} \mathrm{C}\right)$. CLP was performed as described previously (23). Briefly, the rat abdomen was shaved before a $2 \mathrm{~cm}$ long midline abdominal incision was made. In order to prevent bowel obstruction, the cecum was carefully isolated and $\sim 60 \%$ of the total cecum length was ligated below the ileocecal valve. Two punctures were made on the opposite side of the mesenteric with a sterile 18-gauge needle. The cecum was then gently pressed to expel a small amount of stool from the puncture site to ensure a full-thickness perforation. The cecum was returned to the peritoneal cavity, and the abdominal incision was closed in two layers. The cecums of sham-operated rats were not ligated or punctured, but underwent the same procedure as aforementioned. All rats were subcutaneously injected with normal saline $(5 \mathrm{ml} / 100 \mathrm{~g})$ immediately following surgery in order to resuscitate. They were then group-housed in a temperature-controlled room $\left(22^{\circ} \mathrm{C}\right)$ in cages with dry sawdust bedding. Fluid blocks and soft food were provided. The mortality and behavioral signs were monitored and recorded every day following the CLP procedure.
To identify the effect of apelin on sepsis-induced cardiomyopathy, apelin ( $2 \mathrm{mg} / \mathrm{kg} /$ day; American Peptide, Sunnyvale, CA, USA) or an equal dose of normal saline were administered daily intraperitoneally after the sepsis model was induced for 3 days. Each group was comprised of 10 rats. Seven days post-surgery, the heart and blood from the rats were collected for subsequent experiments.

ELISA. Plasma inflammatory factors, interleukin (IL)- $1 \beta$ (cat. no. RAB0273), IL-6 (cat. no. RAB0306), tumor necrosis factor (TNF)- $\alpha$ (cat. no. RAB1089), and apelin, were measured with commercially available ELISA kits (Sigma-Aldrich; Merck KGaA). All procedures were performed according to the manufacturer's instructions.

Immunohistochemical analysis. Myocardial tissue samples were fixed in $10 \%$ formalin solution prior to embedding in paraffin. The $4 \mu \mathrm{m}$ thickness sections were stained with hematoxylin and eosin (H\&E) or terminal deoxynucleotidyl transferase (TdT) dUTP nick-end labeling (TUNEL) at room temperature. Nuclear were stain with DAPI. The results were observed using an Axiophot light microscope (Zeiss AG, Oberkochen, Germany) and photographed with a digital camera. A total of 10 random fields in each group were selected for statistical analysis.

Western blot analysis. Western blot analyses for TLR4 and NLRP3 signaling pathway proteins in myocardial tissues were performed as previously described (24). Briefly, proteins in myocardial tissue lysates were separated by electrophoresis and transferred to a polyvinylidene difluoride membrane. The membranes were blocked and then incubated with antibodies against GAPDH (cat. no. G5262; 1:200), NLRP3 (cat. no. HPA012878; 1:200), ASC (cat. no. SAB4501315; 1:200), caspase-1 (cat. no. C5482; 1:200), TLR4 (cat. no. SAB1301541;

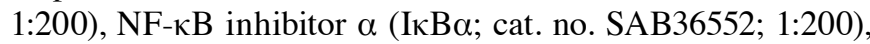
phosphorylated (p)-IкB $\alpha$ (cat. no. SAB625455; 1:200), RELA proto-oncogene NF- $\mathrm{B}$ subunit (also known as P65) (cat. no. SAB251154; 1:200), and p-P65 (all from Sigma-Aldrich; Merck KGaA) (cat.no. SAB4536551; 1:200), in TBST buffer overnight at $4^{\circ} \mathrm{C}$. The membranes were then incubated with secondary antibody linked to horseradish peroxidase (Invitrogen; Thermo Fisher Scientific, Inc., Waltham, MA, USA). Immunoreactive bands were developed using an enhanced chemiluminescence reagent (Pierce; Thermo Fisher Scientific, Inc.). Band densities were normalized to the density of GAPDH and quantified with scanning densitometric analysis using ImageJ software (National Institutes of Health, Bethesda, MD, USA).

Statistical analysis. All data are presented as the mean \pm standard deviation. Differences between treatment groups were analyzed using one-way analysis of variance followed by Tukey's multiple comparisons test, using GraphPad Prism 6.0 software (GraphPad Software Inc., La Jolla, CA, USA). $\mathrm{P}<0.05$ was considered to indicate a statistically significant difference.

\section{Results}

Inflammatory factors and cardiac functional analyses in patients with SICM. In total, 73 participants with or without 
Table I. Comparison of the clinicopathological characteristics in SICM patients and healthy controls.

\begin{tabular}{|c|c|c|c|}
\hline Clinical parameter & SICM & Healthy control & P-value \\
\hline \multicolumn{4}{|l|}{ Sex } \\
\hline Male & $26(66.7 \%)$ & $20(58.8 \%)$ & \\
\hline Female & $13(33.3 \%)$ & $14(41.2 \%)$ & \\
\hline Age & $71.6 \pm 10.7$ & $51.6 \pm 15.9$ & 0.0188 \\
\hline HR (bpm) & $65.6 \pm 7.7$ & $76.4 \pm 8.7$ & 0.082 \\
\hline $\mathrm{EF}(\%)$ & $32.8 \pm 4.6$ & $57.7 \pm 6.6$ & 0.003 \\
\hline $\mathrm{FS}(\%)$ & $21.6 \pm 3.2$ & $30.1 \pm 4.7$ & 0.005 \\
\hline LVESD (mm) & $29.3 \pm 2.8$ & $33.2 \pm 7.0$ & 0.067 \\
\hline LVEDD (mm) & $37.2 \pm 4.4$ & $46.7 \pm 5.7$ & 0.023 \\
\hline $\mathrm{SV}(\mu 1)$ & $24.6 \pm 3.6$ & $57.7 \pm 13.1$ & 0.0032 \\
\hline Long of ICU Stay (days) & $23.0 \pm 22.9$ & 0 & - \\
\hline 28 d survival (days) & $23(59.0 \%)$ & - & - \\
\hline PCT (ng/ml) & $3.5 \pm 3.1$ & $78.6 \pm 58.6$ & 0.0001 \\
\hline WBC $\left(\times 10^{9} / 1\right)$ & $15.5 \pm 10.2$ & $6.5 \pm 1.1$ & 0.0001 \\
\hline $\mathrm{CRP}(\mathrm{mg} / \mathrm{dl})$ & $128.1 \pm 54.4$ & $8.7 \pm 11.2$ & 0.0001 \\
\hline \multicolumn{4}{|l|}{ Blood culture } \\
\hline $\mathrm{G}(+)$ & $5(7.7 \%)$ & 0 & - \\
\hline $\mathrm{G}(-)$ & $23(59.0 \%)$ & 0 & - \\
\hline Fungus & $7(17.9 \%)$ & & \\
\hline \multicolumn{4}{|l|}{ Sources of infection } \\
\hline Lower respiratory tract & $26(66.7 \%)$ & 0 & - \\
\hline Upper respiratory tract & 0 & 0 & - \\
\hline Urinary tract & $2(5.1 \%)$ & 0 & - \\
\hline Intra-abdominal & $2(5.1 \%)$ & 0 & - \\
\hline Musculoskeletal/soft tissue & $5(12.8 \%)$ & 0 & - \\
\hline Intravascular & $1(2.6 \%)$ & 0 & - \\
\hline \multicolumn{4}{|l|}{ Inflammatory cytokines } \\
\hline IL-6 (pg/ml) & $63.5 \pm 25.5$ & $14.8 \pm 6.5$ & 0.001 \\
\hline $\mathrm{IL}-1 \beta(\mathrm{pg} / \mathrm{ml})$ & $124.6 \pm 84.1$ & $22.5 \pm 11.6$ & 0.001 \\
\hline $\mathrm{TNF}-\alpha(\mathrm{pg} / \mathrm{ml})$ & $22.8 \pm 16.6$ & $5.6 \pm 3.5$ & 0.001 \\
\hline
\end{tabular}

Data are expressed as the mean \pm standard deviation. SICM, sepsis-induced cardiomyopathy; HR, heart rate; EF, ejection fraction; FS, fractional shortening; LVESD, left ventricular end-systolic dimension; LVEDD, left ventricular end-diastolic dimension; SV, stroke volume; ICU, intensive care unit; PCT, procalcitonin; WBC, white blood cell; CRP, C-reactive protein; G (+), Gram positive; G (-), Gram negative; IL, interleukin; TNF, tumor necrosis factor.

sepsis were studied. Of these, 39 patients had sepsis and 34 subjects were healthy controls. The sex distribution of each group was uniform. In total, 26 patients with SICM were male (66.7\%; Table I). There was a significant difference in age between patients with SICM and those without SICM (Table I). Among the patients with SICM, the 28-day survival in-hospital was $59 \%$ and the average ICU stay was 23 days. Among the patients with SICM, 26 (66.7\%) had respiratory infections, two $(5.1 \%)$ had intra-abdominal infections, two (5.1\%) had urinary tract infections, five $(12.8 \%)$ had musculoskeletal or soft tissue infections, and one $(2.6 \%)$ had an intravascular infection (Table I). The blood culture was positive in 35 patients with SICM; five (7.7\%) had Gram positive bacterial infections, $23(59 \%)$ had Gram negative bacterial infections, and 7 (17.9\%) had fungal infections (Table I).

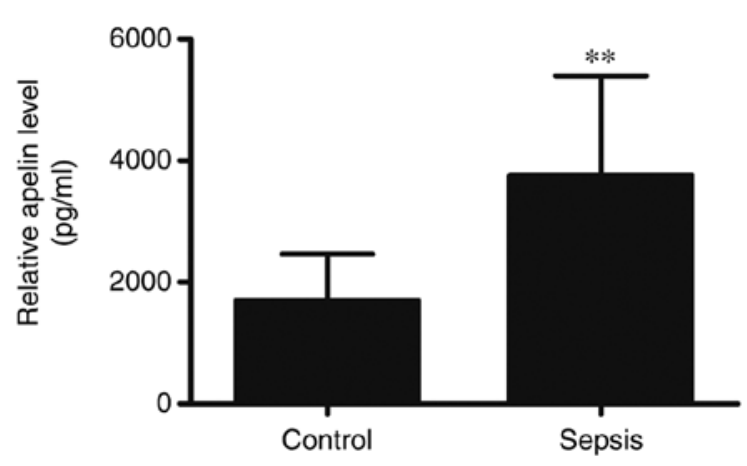

Figure 1. Expression of apelin in plasma from patients with sepsis and from healthy controls. Apelin levels were determined with an ELISA kit. The data are expressed as mean \pm standard deviation. ${ }^{* * *} \mathrm{P}<0.01$ compared with control. 


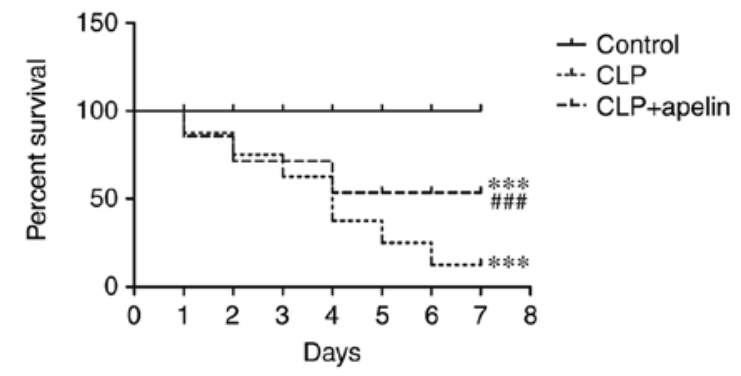

Figure 2. Survival of rats in different groups were analyzed using the Kaplan-Meier method and compared using the log-rank test. The survival of rats treated with apelin $(\mathrm{n}=10)$ was improved compared with the untreated CLP group ( $\mathrm{n}=10)$ within 10 days post-surgery (70 vs. $30 \%$, respectively). Sham-operated rats were used as control. The data are expressed as the mean \pm standard deviation. ${ }^{* * *} \mathrm{P}<0.001$ vs. control; ${ }^{\# \# "} \mathrm{P}<0.001$ vs. CLP group. CLP, cecal ligation and puncture.

Cardiac functional analyses revealed that, compared with healthy controls, fractional shortening (FS), EF, stroke volume (SV), left ventricular end-diastolic dimension (LVEDD) and left ventricular end-systolic dimension (LVESD) were significantly decreased in SICM patients, but the heart rate have no significant difference between the two groups (Table I).

Both C-reactive and white blood cells, which are biomarkers used in the diagnosis of sepsis and inflammation response in SICM patients (25), were significantly increased in SICM patients compared with healthy controls (Table I), suggesting an inflammatory response in SICM patients. ELISA analyses confirmed that plasma levels of IL-6, TNF- $\alpha$ and IL- $1 \beta$ were significantly increased following sepsis (Table I), compared with healthy controls. Apelin, which has anti-inflammatory effects, was also significantly increased in SICM (Fig. 1), suggesting that the expression of apelin might have had a protective effect against SICM.

Apelin treatment improves survival and attenuates CLP sepsis-induced myocardial injury. To determine the protective effects of apelin on cardiac functions during sepsis, apelin $(2 \mathrm{mg} / \mathrm{kg} /$ day $)$ was intraperitoneally administered for 3 consecutive days post-surgery, and the mortality $\%$ was monitored every day for 7 days before the rats were euthanized. The results demonstrated no deaths in the control sham-operated group. Apelin treatment attenuated the mortality of CLP-induced sepsis rats by $30-70 \%$, demonstrating that apelin treatment increased the survival of these animals (Fig. 2).

To identify pathological changes, myocardial tissue was collected for H\&E and TUNEL staining at 7 days post-surgery. Fig. 3A illustrates that the cell structures in the hearts of control sham-operated rats were normal. However, the heart tissues of rats from the CLP group, at seven days post-CLP, exhibited blurring of myocardial stripes, myocardial fiber disarray, unclear cell boundary, small necrosis focus, inflammatory cell infiltration and interstitial edema (Fig. 3A). These pathological changes were partially decreased following apelin treatment (Fig. 3A). Previous studies have reported that sepsis-induced cardiac dysfunction was positively correlated with myocardial apoptosis $(26,27)$. Thus, the TUNEL assay was used to examine the rate of myocardial apoptosis in the experimental groups. As illustrated in Fig. 3B, a marked increase in apoptotic cells was observed in the heart tissues of the CLP rats compared with the control sham-operated rats, confirming that CLP sepsis significantly increased myocardial apoptosis. The numbers of apoptotic cells were obviously decreased with apelin treatment (Fig. 3B), further confirming that apelin had a protective effect on myocardial tissue.

Apelin treatment attenuates the sepsis-induced production of inflammatory cytokines by suppressing TLR4 and $N L R P 3$ signaling. Inflammatory cytokines, such as IL-1 $\beta$, IL- 6 and TNF $\alpha$, have been reported to suppress myocardial function (28). Thus, the effect of apelin on CLP-induced and sepsis-mediated inflammatory cytokine production was examined. Fig. 4 demonstrates that the expression of inflammatory cytokines IL-1 $\beta$, IL- 6 and TNF $\alpha$ were significantly increased following CLP-induced sepsis compared with the sham controls. By contrast, apelin treatment significantly reduced IL-1 $\beta$, TNF $\alpha$ and IL-6 levels in plasma (Fig. 4).

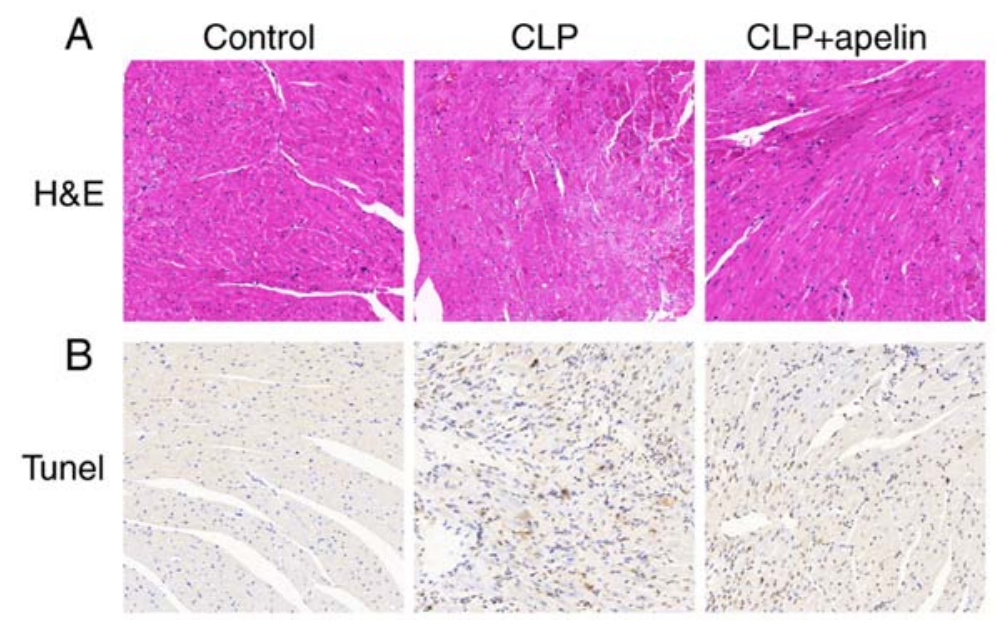

Figure 3. Effects of apelin treatment on histopathological changes in myocardial tissue. (A) Representative images from H\&E staining of heart tissue sections from the three experimental groups. Magnification, x200. (B) Myocardial apoptosis was measured using TUNEL staining of the heart tissue sections. Magnification, $\mathrm{x} 200$. H\&E, hematoxylin and eosin; TUNEL, terminal deoxynucleotidyl transferase dUTP nick-end labeling; CLP, cecal ligation and puncture. 

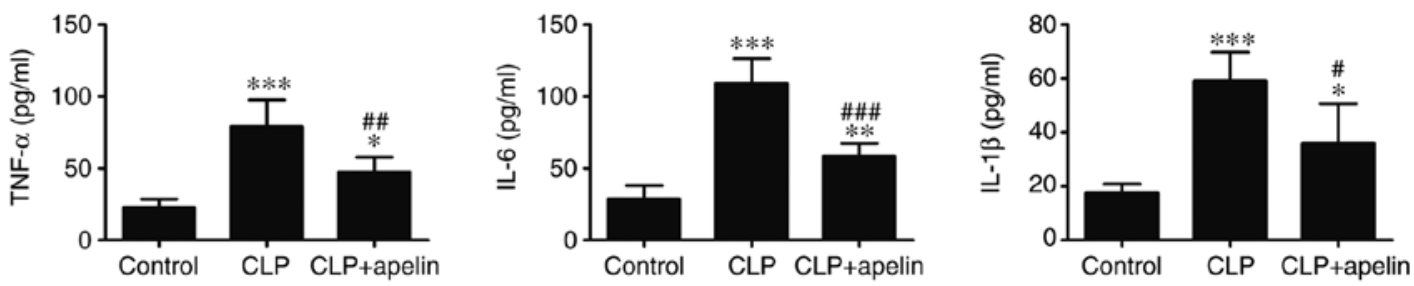

Figure 4. Effects of apelin treatment on sepsis-mediated inflammatory cytokine production. The levels of TNF- $\alpha$, IL- 6 and IL-1 $\beta$ were measured in the plasma of the experimental rats by ELISA. The data are expressed as the mean \pm standard deviation. ${ }^{*} \mathrm{P}<0.05,{ }^{, *} \mathrm{P}<0.01$ and ${ }^{* * * *} \mathrm{P}<0.001$ compared with control; ${ }^{*} \mathrm{P}<0.05$, ${ }^{\# \#} \mathrm{P}<0.01$ and ${ }^{\# \# \#} \mathrm{P}<0.001$ compared with CLP group. TNF, tumor necrosis factor; IL, interleukin; CLP, cecal ligation and puncture.
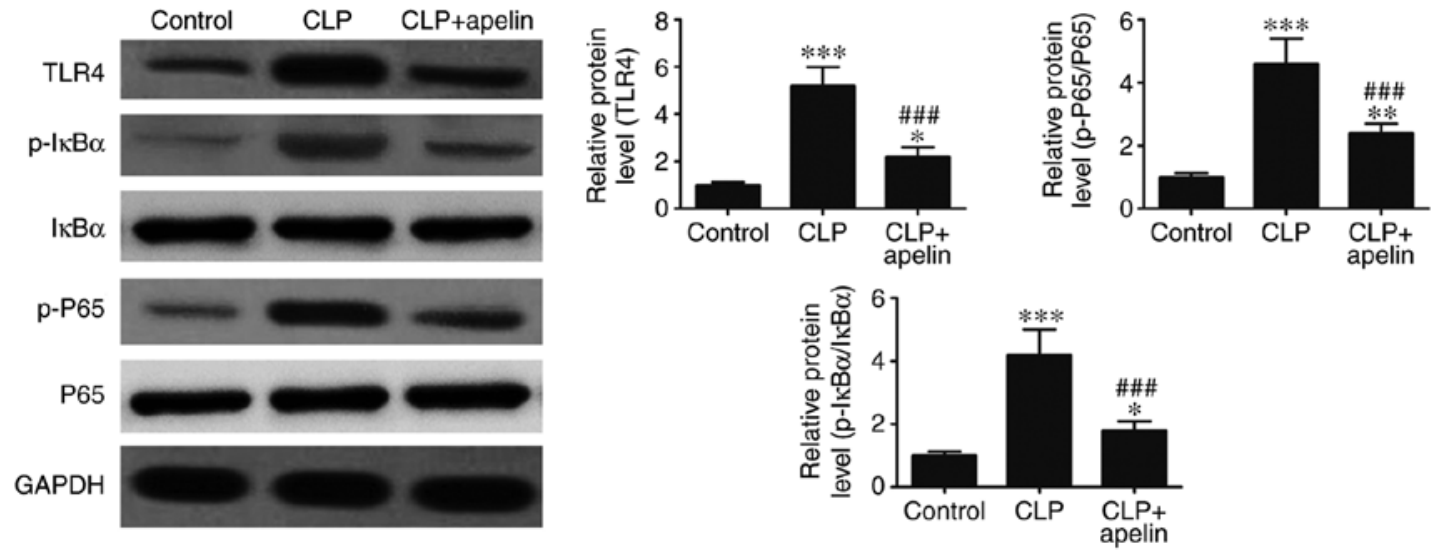

Figure 5. Apelin treatment inhibits the sepsis-induced TLR4 expression and NF-кB activation. The protein expression levels of TLR4 signaling pathway-related proteins were analyzed by western blotting in tissues from the three experimental groups. Representative blots and quantification of relative protein levels are shown. GAPDH was used as a loading control. The data are expressed as the mean \pm standard deviation. ${ }^{*} \mathrm{P}<0.05,{ }^{* *} \mathrm{P}<0.01$ and ${ }^{* * * *} \mathrm{P}<0.001$ compared with control; ${ }^{\# \# \# ~} \mathrm{P}<0.001$ compared with CLP group. TLR4, Toll-like receptor 4; NF, nuclear factor; CLP, cecal ligation and puncture; p-, phosphorylated; IKB $\alpha$,

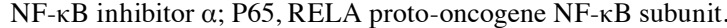
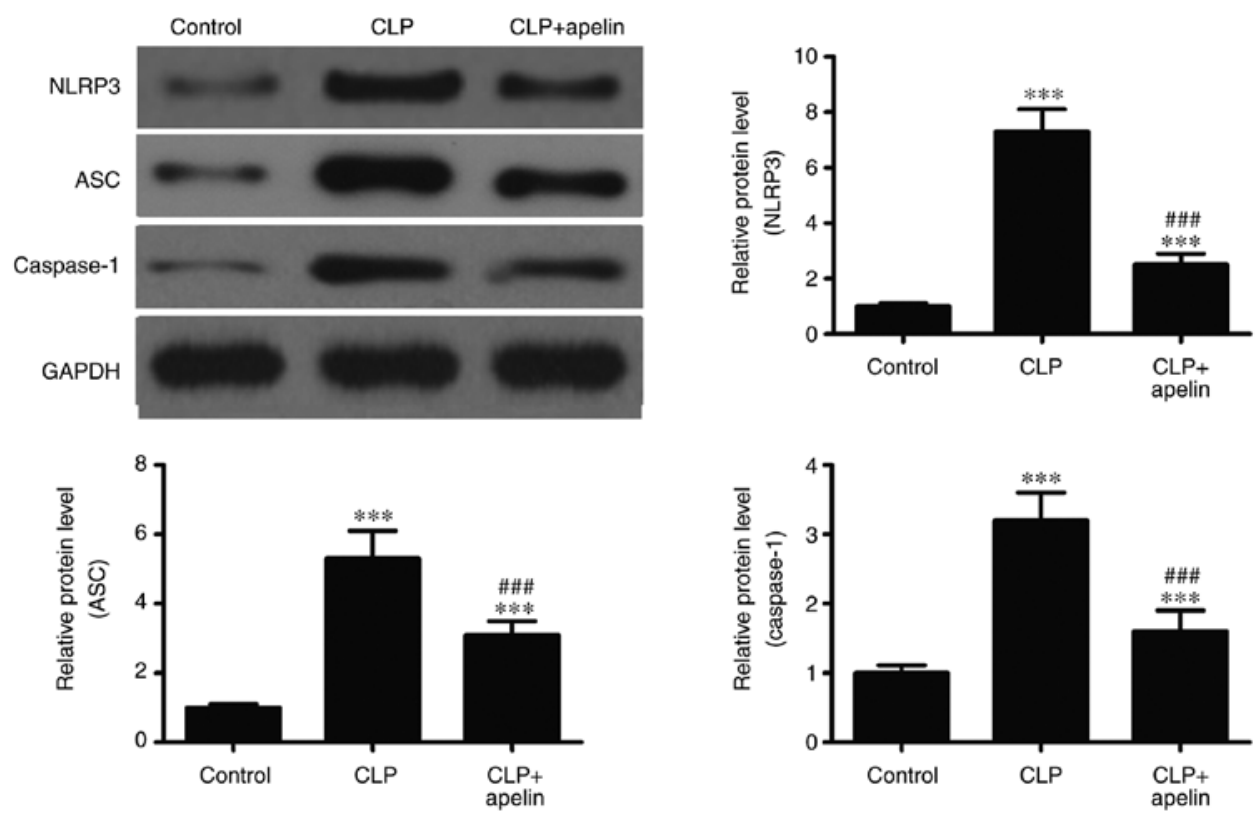

Figure 6. Apelin treatment inhibits sepsis-induced NLRP3 activation. The expression levels of the NLRP3 inflammasome-related proteins were analyzed by western blotting in tissues from the three experimental groups. Representative blots and quantification of relative protein levels are shown. GAPDH was used as a loading control. The data are expressed as the mean \pm standard deviation. ${ }^{* * *} \mathrm{P}<0.001$ compared with control; ${ }^{\# \# \# ~} \mathrm{P}<0.001$ compared with CLP group. NLRP3, NLR family pyrin domain containing 3; ASC, apoptosis-associated speck-like protein; CLP, cecal ligation and puncture.

In order to determine the possible mechanism and relationship between CLP sepsis-induced inflammatory responses and apelin, TLR4/NF-kB pathway-related proteins were analyzed by western blotting. Fig. 5 demonstrates that, compared with 
the CLP group, apelin treatment significantly reversed the sepsis-induced upregulation of TLR4, and the sepsis-induced phosphorylation of P65, and $\mathrm{I} \kappa \mathrm{B} \alpha$.

The present study demonstrated that apelin treatment reduced the IL-1 $\beta$, IL-6 and TNF- $\alpha$ expression in CLP-induced sepsis rats. A previous study has reported that the inactive cytoplasmic precursors pro-IL-1 $\beta$, pro-IL- 6 and pro-TNF- $\alpha$ can be cleaved into their active forms by caspase-1 (12). The NLRP3 inflammasome also serves an important role in caspase-1 activation. Therefore, in order to determine the role of the NLRP3 inflammasome in the inflammatory responses of the myocardium, the levels of NLRP3 inflammasome-related proteins were analyzed by western blotting. The results demonstrated that ASC, NLRP3 and caspase-1 protein expression levels were significantly increased following sepsis, but apelin treatment significantly reversed these effects (Fig. 6). Taken together, the present results suggested that apelin inhibited the expression of inflammatory cytokines by suppressing TLR4 and NLRP3 inflammasome signaling.

\section{Discussion}

The present study revealed that expression of inflammatory cytokines increased in SICM patients who were prone to myocardial dysfunction. The results also revealed the increased expression of plasma apelin in SICM patients, and in vivo experiments demonstrated that apelin treatment reduced the heart damage induced by CLP-mediated sepsis in a rat model. Apelin treatment decreased the plasma levels of IL-6, TNF- $\alpha$ and IL-1 $\beta$, inhibited myocardial apoptosis, and protected against CLP sepsis-induced myocardial dysfunction by inhibiting the TLR4 and NLRP3 signaling pathways.

Inflammatory factors, including IL-6, TNF- $\alpha$ and IL- $1 \beta$, have been recognized to have critical roles in the pathogenesis of CLP-induced sepsis $(29,30)$. Several studies have reported that IL- 6 , TNF- $\alpha$ and IL- $1 \beta$ are involved in myocardial dysfunction $(31,32)$. Furthermore, previous studies have demonstrated that inhibition of the expression of these inflammatory cytokines had a protective effect against SICM $(9,33,34)$. The effects of apelin on inflammatory cytokine production were investigated in the present study. The results demonstrated that apelin protected against CLP sepsis-induced myocardial dysfunction by inhibiting inflammatory cytokine production.

The TLR4 signaling pathway has an important role in pathogenesis of sepsis, which subsequently induces $N F-\kappa B$ activation and releases inflammatory cytokines. Previous studies have reported that sepsis-induced cardiomyopathy is induced through the TLR4 signaling pathway $(35,36)$. A variety of inflammatory genes are regulated by transcriptional factor $\mathrm{NF}-\kappa \mathrm{B}$ (37), and a previous study has demonstrated that the development of cardiomyopathy is closely associated with $N F-\kappa B$ activation (9). In addition, the activation of $N F-\kappa B$ was observed in a CLP-induced rat sepsis model $(38,39)$. The present results suggested a role of apelin in anti-inflammatory responses and revealed an association between apelin and the TLR4 signaling pathway. The present results demonstrated that CLP sepsis-induced TLR4 expression and NF- $\kappa$ B activation were inhibited following apelin treatment. The present study also demonstrated that apelin treatment significantly inhibited NLRP3 inflammasome-induced myocardial dysfunction. NLRP3 is a multiprotein complex, which can promote the secretion of IL-1 $\beta$ by activating caspase-1 (40). A previous study has demonstrated that inhibition of NLRP3 inflammasome expression attenuated the adverse factor-induced myocardial remodeling (41). In conclusion, the findings of the present study provided evidence that apelin may have protective effects against SICM, most likely through the inhibition of the TLR4 and NLRP3 signaling pathways.

\section{Acknowledgements}

Not applicable.

\section{Funding}

This work was funded by grants from the Shanghai Pudong New Area Science and Technology Development Fund Innovative Funding (Health) Program (grant no. PKJ2015-Y55) and the Shanghai Pudong New Area Health System Key Specialty Construction Project (grant no. PWZzk2017-05).

\section{Availability of data and materials}

The analyzed datasets generated during the study are available from the corresponding author on reasonable request.

\section{Author's contributions}

QL, GL, GC and DG generated and analyzed the data. LX, MH and MJ designed the experiments and drafted the manuscript. All authors approved the final version of the manuscript.

\section{Ethics approval and consent to participate}

All experiments involving human subjects or animals were approved by the Human Ethics Committee of Pudong New Area Gongli Hospital (Shanghai, China) and the Animal Ethics Committee of Pudong New Area Gongli Hospital (Shanghai, China), respectively. Informed consent was obtained from all individual participants included in the study.

\section{Consent for publication}

Not applicable.

\section{Competing interests}

The authors declare that they have no competing interests.

\section{References}

1. Papadopoulos P, Pistiki A, Theodorakopoulou M, Christodoulopoulou T, Damoraki G, Goukos D, Briassouli E, Dimopoulou I, Armaganidis A, Nanas S, et al: Immunoparalysis: Clinical and immunological associations in SIRS and severe sepsis patients. Cytokine 92: 83-92, 2017.

2. Alam N, de Ven PM, Oskam E, Stassen P, Kramer MH, Exter PV and Nanayakkara PW: Study protocol for a multi-centre, investigator-initiated, randomized controlled trial to compare the effects of prehospital antibiotic treatment for sepsis patients with usual care after training emergency medical services (EMS) personnel in early recognition (- The prehospital antibiotics against sepsis (PHANTASi) trial. Acute Med 15: 176-184, 2016. 
3. Li L, Hu BC, Chen CQ, Gong SJ, Yu YH, Dai HW and Yan J: Role of mitochondrial damage during cardiac apoptosis in septic rats. Chin Med J 126: 1860-1866, 2013.

4. Ndongson-Dongmo B, Heller R, Hoyer D, Brodhun M, Bauer M, Winning J, Hirsch E, Wetzker R, Schlattmann P and Bauer R: Phosphoinositide 3-kinase gamma controls inflammation-induced myocardial depression via sequential cAMP and iNOS signalling. Cardiovasc Res 108: 243-253, 2015

5. Kalbitz M, Fattahi F, Grailer JJ, Jajou L, Malan EA, Zetoune FS, Huber-Lang M, Russell MW and Ward PA: Complement-induced activation of the cardiac NLRP3 inflammasome in sepsis. FASEB J 30: 3997-4006, 2016.

6. Kakihana Y, Ito T, Nakahara M, Yamaguchi K and Yasuda T: Sepsis-induced myocardial dysfunction: Pathophysiology and management. J Intensive Care 4: 22, 2016.

7. Sato R and Nasu M: A review of sepsis-induced cardiomyopathy. J Intensive Care 3: 48, 2015.

8. Hinkelbein J, Kalenka A, Schubert C, Peterka A and Feldmann RE Jr: Proteome and metabolome alterations in heart and liver indicate compromised energy production during sepsis. Protein Pept Lett 17: 18-31, 2010.

9. Zheng G, Pan M, Jin W, Jin G and Huang Y: MicroRNA-135a is up-regulated and aggravates myocardial depression in sepsis via regulating $\mathrm{p} 38 \mathrm{MAPK} / \mathrm{NF}-\kappa \mathrm{B}$ pathway. Int Immunopharmacol 45: 6-12, 2017.

10. Turillazzi E, Fineschi V, Palmiere $C$ and Sergi C: Cardiovascular involvement in sepsis. Mediators Inflamm 2016: 8584793, 2016.

11. Song E, Jahng JW, Chong LP, Sung HK, Han M, Luo C, Wu D, Boo S, Hinz B, Cooper MA, et al: Lipocalin-2 induces NLRP3 inflammasome activation via HMGB1 induced TLR4 signaling in heart tissue of mice under pressure overload challenge. Am J Transl Res 9: 2723-2735, 2017.

12. Zhang X, Du Q, Yang Y, Wang J, Dou S, Liu C and Duan J: The protective effect of Luteolin on myocardial ischemia/reperfusion (I/R) injury through TLR4/NF- $\mathrm{B}$ /NLRP3 inflammasome pathway. Biomed Pharmacother 91: 1042-1052, 2017.

13. Hao H, Cao L, Jiang C, Che Y, Zhang S, Takahashi S, Wang G and Gonzalez FJ: Farnesoid X receptor regulation of the NLRP3 inflammasome underlies cholestasis-associated sepsis. Cell Metab 25: 856-867 e855, 2017.

14. Zhang W, Tao A, Lan T, Cepinskas G, Kao R, Martin CM and Rui T: Carbon monoxide releasing molecule-3 improves myocardial function in mice with sepsis by inhibiting NLRP3 inflammasome activation in cardiac fibroblasts. Basic Res Cardiol 112: 16, 2017

15. Jin L, Batra S and Jeyaseelan S: Deletion of Nlrp3 augments survival during polymicrobial sepsis by decreasing autophagy and enhancing phagocytosis. J Immunol 198: 1253-1262, 2017.

16. Kim MJ, Yoon JH and Ryu JH: Mitophagy: A balance regulator of NLRP3 inflammasome activation. BMB Rep 49: 529-535, 2016.

17. Shao QH, Zhang XL, Yang PF, Yuan YH and Chen NH: Amyloidogenic proteinsassociated with neurodegenerativediseases activate the NLRP3 inflammasome. Int Immunopharmacol 49: $155-160,2017$.

18. Awad F, Assrawi E, Jumeau C, Georgin-Lavialle S, Cobret L, Duquesnoy P, Piterboth W, Thomas L, Stankovic-Stojanovic K, Louvrier C, et al: Impact of human monocyte and macrophage polarization on NLR expression and NLRP3 inflammasome activation. PLoS One 12: e0175336, 2017.

19. Han S, Englander EW, Gomez GA and Greeley GH Jr: Apelin regulates nuclear factor- $\kappa B^{\prime} s$ involvement in the inflammatory response of pancreatitis. Pancreas 46: 64-70, 2017.

20. Luo K, Long H, Xu B and Luo Y: Apelin attenuates postburn sepsis via a phosphatidylinositol 3-kinase/protein kinase B dependent mechanism: A randomized animal study. Int J Surg 21: 22-27, 2015.

21. Zhang P, Yi LH, Meng GY, Zhang HY, Sun HH and Cui LQ Apelin-13 attenuates cisplatin-induced cardiotoxicity through inhibition of ROS-mediated DNA damage and regulation of MAPKs and AKT pathways. Free Radic Res 51: 449-459, 2017.

22. Zhang X, Hu W, Feng F, Xu J and Wu F: Apelin-13 protects against myocardial infarction-induced myocardial fibrosis. Mol Med Rep 13: 5262-5268, 2016.

23. Zhou Q, Pan X, Wang L, Wang X and Xiong D: The protective role of neuregulin-1: A potential therapy for sepsis-induced cardiomyopathy. Eur J Pharmacol 788: 234-240, 2016.
24. Xiao M, Li L, Li C, Zhang P, Hu Q, Ma L and Zhang H: Role of autophagy and apoptosis in wound tissue of deep second-degree burn in rats. Acad Emerg Med 21: 383-391, 2014.

25. Yao L, Liu Z, Zhu J, Li B, Chai C and Tian Y: Clinical evaluation of circulating microRNA-25 level change in sepsis and its potential relationship with oxidative stress. Int J Clin Exp Pathol 8 7675-7684, 2015

26. Zhang M, Wang X, Bai B, Zhang R, Li Y and Wang Y: Oxymatrine protects against sepsis-induced myocardial injury via inhibition of the TNF- $\alpha /$ p38-MAPK/caspase-3 signaling pathway. Mol Med Rep 14: 551-559, 2016.

27. An R, Zhao L, Xi C, Li H, Shen G, Liu H, Zhang S and Sun L: Melatonin attenuates sepsis-induced cardiac dysfunction via a PI3K/Akt-dependent mechanism. Basic Res Cardiol 111: 8, 2016.

28. Zhao H, Zhang M, Zhou F, Cao W, Bi L, Xie Y, Yang Q and Wang S: Cinnamaldehyde ameliorates LPS-induced cardiac dysfunction via TLR4-NOX4 pathway: The regulation of autophagy and ROS production. J Mol Cell Cardiol 101: 11-24, 2016.

29. Lee S, Nakahira K, Dalli J, Siempos II, Norris PC, Colas RA, Moon JS, Shinohara M, Hisata S, Howrylak JA, et al: NLRP3 inflammasome deficiency protects against microbial sepsis via increased lipoxin $B_{4}$ synthesis. Am J Respir Crit Care Med 196: 713-726, 2017.

30. Cho W, Koo JY, Park Y, Oh K, Lee S, Song JS, Bae MA, Lim D, Lee DS and Park SB: treatment of sepsis pathogenesis with high mobility group box protein 1-regulating anti-inflammatory agents. J Med Chem 60: 170-179, 2017.

31. Gao M, Ha T, Zhang X, Liu L, Wang X, Kelley J, Singh K, Kao R, Gao X, Williams D and Li C: Toll-like receptor 3 plays a central role in cardiac dysfunction during polymicrobial sepsis. Crit Care Med 40: 2390-2399, 2012.

32. Ha T, Xia Y, Liu X, Lu C, Liu L, Kelley J, Kalbfleisch J, Kao RL, Williams DL and Li C: Glucan phosphate attenuates myocardial HMGB1 translocation in severe sepsis through inhibiting NF- $\mathrm{B}$ activation. Am J Physiol Heart Circ Physiol 301: H848-H855, 2011.

33. Ma H, Wang X, Ha T, Gao M, Liu L, Wang R, Yu K, Kalbfleisch JH, Kao RL, Williams DL and Li C: MicroRNA-125b prevents cardiac dysfunction in polymicrobial sepsis by targeting TRAF6-mediated nuclear factor $\kappa \mathrm{B}$ activation and $\mathrm{p} 53$-mediated apoptotic signaling. J Infect Dis 214: 1773-1783, 2016.

34. Gao F, Yang YZ, Feng XY, Fan TT, Jiang L, Guo R and Liu Q: Interleukin-27 is elevated in sepsis-induced myocardial dysfunction and mediates inflammation. Cytokine 88: 1-11, 2016.

35. Vaez H, Rameshrad M, Najafi M, Barar J, Barzegari A and Garjani A: Cardioprotective effect of metformin in lipopolysaccharide-induced sepsis via suppression of toll-like receptor 4 (TLR4) in heart. Eur J Pharmacol 772: 115-123, 2016.

36. Rameshrad M, Maleki-Dizaji N, Vaez H, Soraya H, Nakhlband A and Garjani A: Lipopolysaccharide induced activation of toll like receptor 4 in isolated rat heart suggests a local immune response in myocardium. Iran J Immunol 12: 104-116, 2015.

37. Afonina IS, Zhong Z, Karin M and Beyaert R: Limiting inflammation-the negative regulation of NF- $\mathrm{BB}$ and the NLRP3 inflammasome. Nat Immunol 18: 861-869, 2017.

38. Wang N, Mao L, Yang L, Zou J, Liu K, Liu M, Zhang H, Xiao X and Wang K: Resveratrol protects against early polymicrobial sepsis-induced acute kidney injury through inhibiting endoplasmic reticulum stress-activated NF- $\kappa \mathrm{B}$ pathway. Oncotarget 8: 36449-36461, 2017.

39. Chen J, Kieswich JE, Chiazza F, Moyes AJ, Gobbetti T, Purvis GS, Salvatori DC, Patel NS, Perretti M, Hobbs AJ, et al: I $\mathrm{B}$ kinase inhibitor attenuates sepsis-induced cardiac dysfunction in CKD. J Am Soc Nephrol 28: 94-105, 2017.

40. Tschopp J and Schroder K: NLRP3 inflammasome activation: The convergence of multiple signalling pathways on ROS production? Nat Rev Immunol 10: 210-215, 2010.

41. Li R, Lu K, Wang Y, Chen M, Zhang F, Shen H, Yao D, Gong K and Zhang Z: Triptolide attenuates pressure overload-induced myocardial remodeling in mice via the inhibition of NLRP3 inflammasome expression. Biochem Biophys Res Commun 485: $69-75,2017$ 